\title{
Preparation and Characterization of IPN Microspheres for Controlled Delivery of Naproxen
}

\author{
Ebru Kondolot Solak
}

Department of Chemistry and Chemical Processing Technology, Atatürk Vocational High School, Gazi University, Ankara, Turkey. Email: ebrukondolot@gazi.edu.tr

Received July $14^{\text {th }}$, 2011; revised August 22 ${ }^{\text {nd }}, 2011$; accepted September $8^{\text {th }}, 2011$.

\begin{abstract}
Interpenetrating network (IPN) microspheres of sodium alginate (NaAlg) and poly (vinyl alcohol) (PVA) were prepared and crosslinked with glutaraldehyde (GA) by using the water in oil (W/O) emulsification method to deliver naproxen sodium (NS). NS was successfully encapsulated into IPN microspheres in different ratios of NaAlg and PVA (w/w), drug loading percentage $(w / w)$ and crosslinking time. Crosslink density of the matrices was affected by the time of crosslinker. The prepared microspheres were characterized by Fourier transform infrared spectroscopy (FTIR). Pictures of selected microspheres were determined using optic microscope. Results confirmed the dispersion of NS in the microspheres. Release of NS from the microspheres was investigated in $\mathrm{pH}$ 1.2, 6.8 and 7.4 media for two hours respectively. The highest NS release was obtained as $92 \%(w / w)$ by using UV spectroscopy. Equilibrium swelling was performed in $p H 7.4$ buffer solution at $37^{\circ} \mathrm{C}$.
\end{abstract}

Keywords: Controlled Release, Microspheres, Alginate

\section{Introduction}

IPN is a polymer comprising two or more networks which are at least partially interlaced on a polymer scale but not covalently bonded to each other. The network cannot be separated unless chemical bonds are broken. The two or more networks can be envisioned to be entangled in such a way that they are concatenated and cannot be pulled apart, but not bonded to each other by any chemical bond [1,2].

Controlled release technology has important potential in the fields of medicine, pharmacy and agriculture. In these areas natural polymeric materials have been preferred over synthetic polymers due to their low cost, nontoxicity, easy availability and biodegradability properties [3-5]. Biodegradable polymers derived from NaAlg and PVA have shown to be useful in pharmaceutical industries due to their ability for drug release [6-8]. Sodium Alginate (NaAlg) is a biodegradable polymer that has been widely used in controlled release applications of pesticides [9-12] and drugs [13,14].

Poly(vinyl alcohol) (PVA) is also a suitable polymer for drug release because of its desirable properties such as nontoxicity and noncarcinogenicity and has been used in many studies due to its biocompatibility. However it is difficult to prepare beads from this polymer due to its poor stability. A blending technique can be considered as a useful tool for the preparation of new alginate beads with PVA to increase the bead forming ability in aqueous medium. PVA can strongly interact with NaAlg through hydrogen bonding on a molecular level. For this reason in several studies, NaAlg and PVA were chosen for the microsphere formation and successfully crosslinked with glutaraldehyde $[15,16]$.

Naproxen Sodium (NS) is a non-steroidal and anti-inflammatory drug with analgesic properties however gastrointestinal side effects such as bleeding, ulceration or perforation were commonly seen when this drug was used. For this reason it is important to obtain prolonged or controlled drug delivery to improve bioavailability or stability and to target the drug to a specific site.

According to our literature survey there is no report available about the formation of IPN structure of PVA with NaAlg for the controlled release of NS in $\mathrm{pH} 1.2$, 6.8 and 7.4 medium. The present investigation is related to the in vitro release studies on IPN microspheres formulations loaded with different amounts of NS. Release characteristics of the formulations were studied for their exposure time to cross-linking agent, at different amounts of NS and polymers.

\section{Materials and Methods}

\subsection{Materials}

NaAlg (medium viscosity) was purchased from Sigma 
Chemical Co. (St. Louis, MO). PVA was procured by Merck (Darmstadt, Germany). The molecular weight and degree of saponification of PVA were 72000 and greater than $98 \%$, respectively. Span-85, GA (25\% w/w), hydrochloric acid, light liquid paraffin, hexane, $\mathrm{Na}_{2} \mathrm{HPO}_{4}$ and $\mathrm{NaH}_{2} \mathrm{PO}_{4}$ used in this study were all supplied by Merck. A gift sample of NS was obtained from Novartis (Summit, NJ).

\subsection{Preparation of the IPN Microspheres and Drug Loading}

IPN microspheres of PVA and NaAlg were prepared by emulsion-crosslinking method and GA was used as a cross linking agent. NaAlg was dissolved separately in distilled water at different concentrations by stirring. After PVA was dispersed in NaAlg solution and stirred overnight to obtain a homogeneous solution, required amount of drug was dispersed in the polymer solution. The drug loaded polymer solution was emulsified into light liquid paraffin to form water-in-oil (W/O) emulsion using a high speed stirrer in a beaker containing light liquid paraffin oil, Span-85 (2\% (w/v)), $0.1 \mathrm{M} \mathrm{HCl}$ and the required amount of GA. The microspheres formed were filtered, washed repeatedly with n-hexane and water to remove the oil as well as excess amount of surfactant and unreacted GA. These microspheres were dried in oven at $40^{\circ} \mathrm{C}$ and stored for further analysis.

The microspheres were prepared with different formulations which were presented at Table 1. A schematic representation of the structure of IPN is given in Figure 1.

\subsection{Fourier Transforms Infrared Spectroscopy (FTIR)}

FTIR spectral measurements were performed with a Mattson 1000 FTIR spectrometer (Welwyn Garden, England) to confirm the presence of crosslinking and drug in PVA/NaAlg.

\subsection{Optical Microscopy Study}

Optical microscope imaging was performed using Leica L2 optic microscope (California, United States).

\subsection{Swelling Studies}

The equilibrium swelling degree of the crosslinked empty IPN microspheres was determined by measuring gravimetrically the extent of their swelling in $\mathrm{pH} 7.4$ buffer solution at $37^{\circ} \mathrm{C}$. To ensure complete equilibration the samples were allowed to swell for $48 \mathrm{~h}$. The excess surface-adhered liquid drops were removed by blotting. The swollen microspheres were weighed using electronic balance (Precisa XB 220 A, USA). The microspheres were then dried in an oven at $40^{\circ} \mathrm{C}$, until there was no

Table 1. Results of percent entrapment efficiency and yield at various crosslinker times.

\begin{tabular}{|c|c|c|c|c|c|}
\hline Formulation Code & Polymers & $\begin{array}{c}\text { \% Naproxen sodium } \\
\text { loaded (w/w) }\end{array}$ & $\begin{array}{l}\text { Time of exposure } \\
\text { to GA (min) }\end{array}$ & $\begin{array}{c}\text { Entrapment } \\
\text { efficiency (\%) }\end{array}$ & Yield (\%) \\
\hline $\mathrm{A} 1$ & NaAlg & 50 & 5 & 61 & 78 \\
\hline A2 & NaAlg & 33 & 5 & 63 & 81 \\
\hline A3 & NaAlg & 20 & 5 & 68 & 77 \\
\hline A4 & NaAlg & 33 & 10 & 58 & 76 \\
\hline A5 & NaAlg & 33 & 15 & 56 & 80 \\
\hline B1 & $\begin{array}{c}\text { PVA } 66 \%(w / w) \\
\text { NaAlg } 33 \%(w / w)\end{array}$ & 50 & 5 & 66 & 89 \\
\hline B2 & $\begin{array}{c}\text { PVA } 66 \%(w / w) \\
\text { NaAlg } 33 \%(w / w)\end{array}$ & 33 & 5 & 60 & 85 \\
\hline B3 & $\begin{array}{c}\text { PVA } 66 \%(\mathrm{w} / \mathrm{w}) \\
\text { NaAlg } 33 \%(\mathrm{w} / \mathrm{w})\end{array}$ & 20 & 5 & 54 & 79 \\
\hline C1 & $\begin{array}{c}\text { PVA } 50 \%(\mathrm{w} / \mathrm{w}) \\
\text { NaAlg } 50 \%(\mathrm{w} / \mathrm{w})\end{array}$ & 50 & 5 & 69 & 86 \\
\hline C2 & $\begin{array}{c}\text { PVA } 50 \%(\mathrm{w} / \mathrm{w}) \\
\text { NaAlg } 50 \%(\mathrm{w} / \mathrm{w})\end{array}$ & 33 & 5 & 64 & 88 \\
\hline C3 & $\begin{array}{c}\text { PVA } 50 \%(\mathrm{w} / \mathrm{w}) \\
\text { NaAlg } 50 \%(\mathrm{w} / \mathrm{w})\end{array}$ & 20 & 5 & 63 & 85 \\
\hline D1 & $\begin{array}{c}\text { PVA } 33 \%(w / w) \\
\text { NaAlg } 66 \%(w / w)\end{array}$ & 50 & 5 & 72 & 83 \\
\hline D2 & $\begin{array}{c}\text { PVA } 33 \%(w / w) \\
\text { NaAlg } 66 \%(w / w)\end{array}$ & 33 & 5 & 67 & 78 \\
\hline D3 & $\begin{array}{c}\text { PVA } 33 \%(w / w) \\
\text { NaAlg } 66 \%(w / w)\end{array}$ & 20 & 5 & 65 & 75 \\
\hline
\end{tabular}




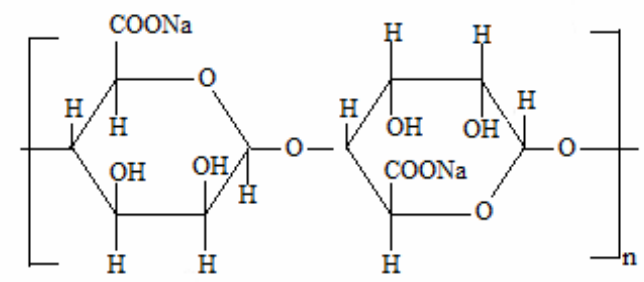<smiles>CCCCCC(=O)CCCCC</smiles>

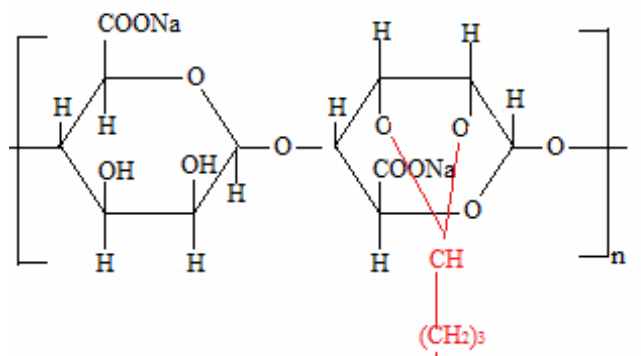

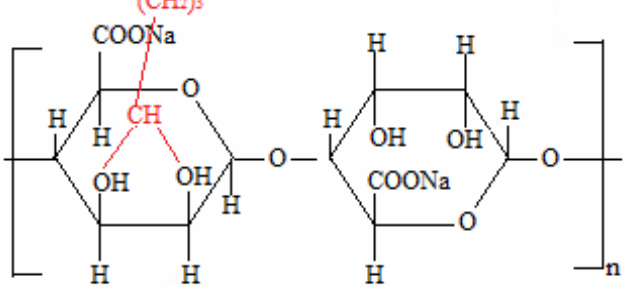<smiles>CCC(O)CC(C)(C)CC</smiles><smiles>[AlH2]</smiles><smiles>O=CCCCC=O</smiles>

GA

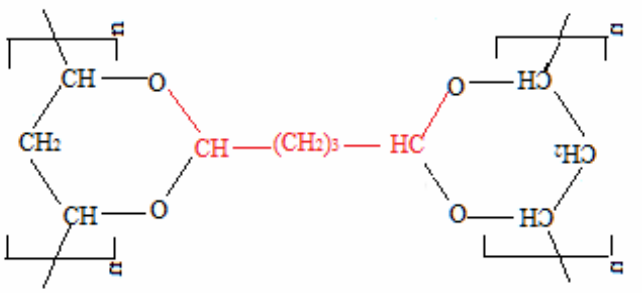

Crosslinked PVA chains

Crosslinked NaAlg chains

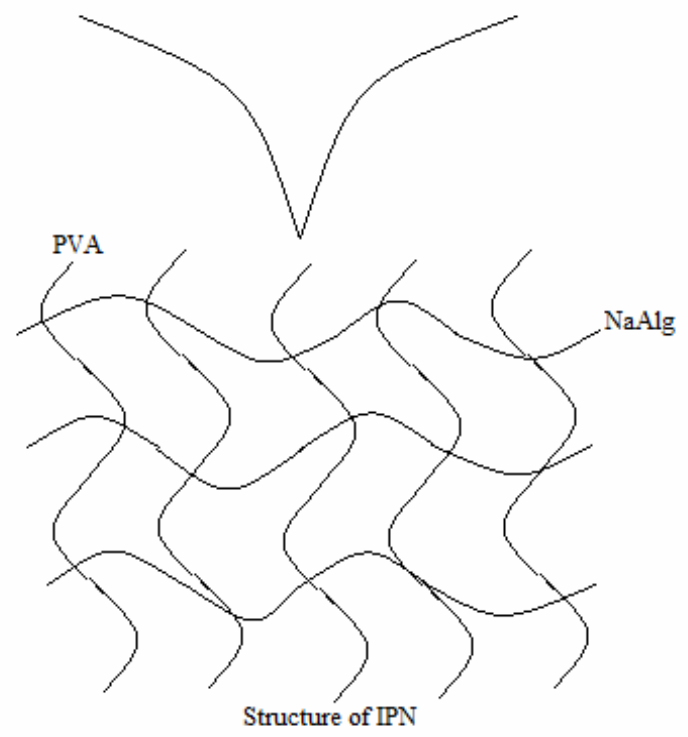

Figure 1. Schematic representation of structure of IPN. 
change in the dried mass of samples. The percent equilibrium swelling degree was calculated as follows:

$$
\text { Equilibrium swelling degree (\%) }=\frac{M_{s}-M_{d}}{M_{d}} \times 100
$$

where $M_{s}$ and $M_{d}$ are the mass of the swollen and dry microspheres, respectively.

\subsection{Entrapment Efficiency}

Required amount of dry microspheres was crushed in an agate mortar with a pestle, stirred with water and refluxed at $25^{\circ} \mathrm{C}$ for $1 \mathrm{~h}$, to ensure the complete extraction of NS from the beads. At the end of the $1 \mathrm{~h}$, precipitated microspheres were filtered and NS was analyzed by using a UV spectrophotometer (Unico 4802 UV/VIS) at a wavelength of $271 \mathrm{~nm}$. The percentage of entrapment efficiency was then calculated as follows:

$$
\text { Entrapment efficiency }(\%)=\frac{\text { Practical loading }}{\text { Theoretical loading }} \times 100
$$

\subsection{In Vitro NS Release}

In vitro drug release from the IPN microspheres was studied at $\mathrm{pH} 1.2 \mathrm{HCl}$ solution, $\mathrm{pH} 6.8$ and $\mathrm{pH} 7.4$ phosphate buffer solutions and incubated in a shaking water bath (Medline BS-21, Korea) at $37^{\circ} \mathrm{C}$. At $2 \mathrm{~h}$ intervals medium was changed to be $\mathrm{pH}: 1.2,6.8$ and 7.4 , respectively. At specific time intervals, the NS content was determined using UV spectrophotometer at $271 \mathrm{~nm}$. Analyzed solution was added back to the dissolution media to maintain a constant volume. From the absorbance values the cumulative released amount percentage was determined. All experiments were performed in triplicate to minimize the variation error. The average values were used for further data treatment and plotting.

\section{Results and Discussion}

\subsection{Characterization of Microspheres}

Results of FTIR spectra for powder NS, NaAlg, PVA, empty IPN microspheres and NS loaded IPN microspheres are shown in Figure 2.

The entire bead formulations showed a broad band between the 3000 and $3500 \mathrm{~cm}^{-1}$, which was attributed to $-\mathrm{OH}$ stretching vibrations. The peak at $1618 \mathrm{~cm}^{-1}$ in the spectrum of NaAlg is due to the stretching band of carbonyl stretching $(\mathrm{C}=\mathrm{O})$. A broad characteristic peak at $1625 \mathrm{~cm}^{-1}$ is due to the $\mathrm{C}=\mathrm{O}$ of the NaAlg polymeric chain and unhydrolyzed part in PVA in the IPN. In the spectrums of NaAlg, PVA and IPN appear stretching bands of C-H group at $2940 \mathrm{~cm}^{-1}, 2986 \mathrm{~cm}^{-1}$ and 2920 $\mathrm{cm}^{-1}$, respectively. As it is seen from Figure 2 the intensity of $-\mathrm{OH}$ peak corresponding to crosslinked IPN is narrower than the uncrosslinked NaAlg and PVA as the evidence of crosslinking.

Optic microscope images of dried NS loaded IPN microspheres were shown in Figure 3. As it was reflected from the figure, microspheres almost maintain their spherical form.

Swelling results were shown in Table 2. Swelling characteristics depends upon the amount of polymer. Equilibrium swelling (\%) increased with increasing amount of PVA in the IPN matrix. Since PVA and NaAlg are water soluble polymers, the swelling of IPN will increase due to their higher water uptake.

\subsection{In Vitro Release Study}

$\%$ Cumulative release results were shown in Figure 4 for $50 \%$ (wt) NS loaded IPN microspheres. The formulations of these microspheres were given in Table $\mathbf{1}$ as A1, B1, C1 and D1. Similarly release results for 33\% (wt) and $20 \%$ (wt) NS loaded microspheres were shown in Figure 5 and Figure 6, respectively. \% 33 NS loaded IPN microspheres were formulated as A2, B2, C2, D2 and $\% 20$ NS loaded IPN microspheres were shown as A3, B3, C3, D3.

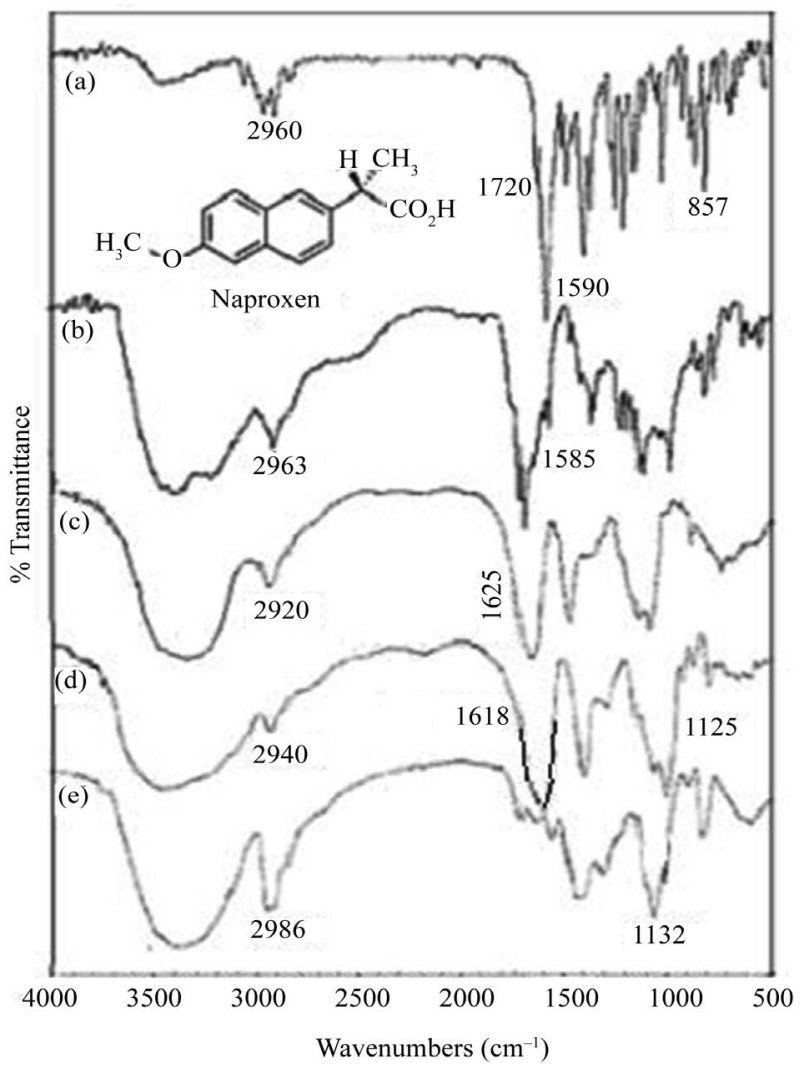

Figure 2. FTIR spectra of (a) NS, (b) NS loaded IPN microspheres, (c) empty IPN microspheres, (d) powder NaAlg (e) powder PVA. 


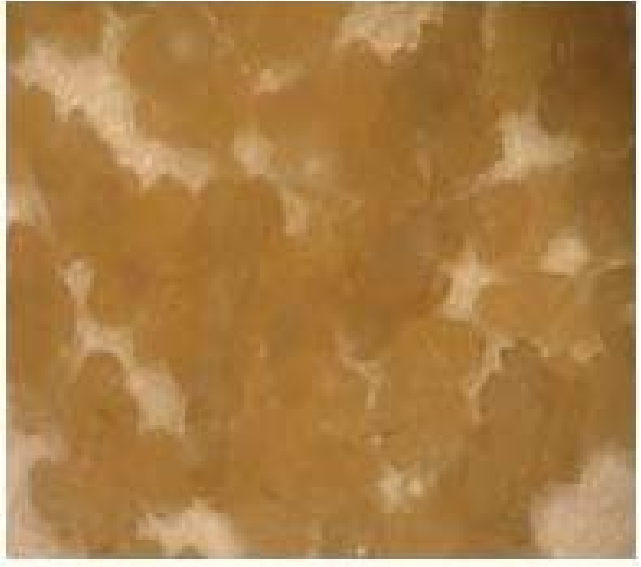

Figure 3. Optic microscope imaging of NS loaded IPN microspheres.

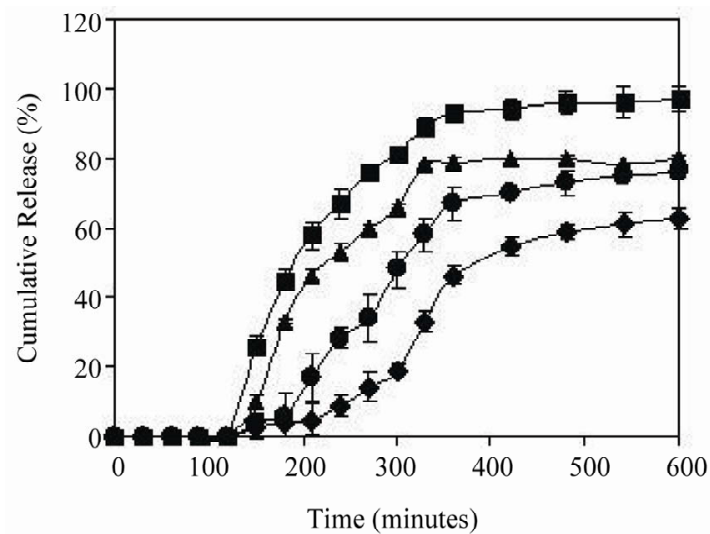

Figure 4. \% Cumulative release of NS from different IPN formulations loaded with $50 \%$ of drug at concentration of GA: 2.5\% and exposure time to GA: $15 \mathrm{~min}$. Symbols: A1

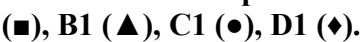

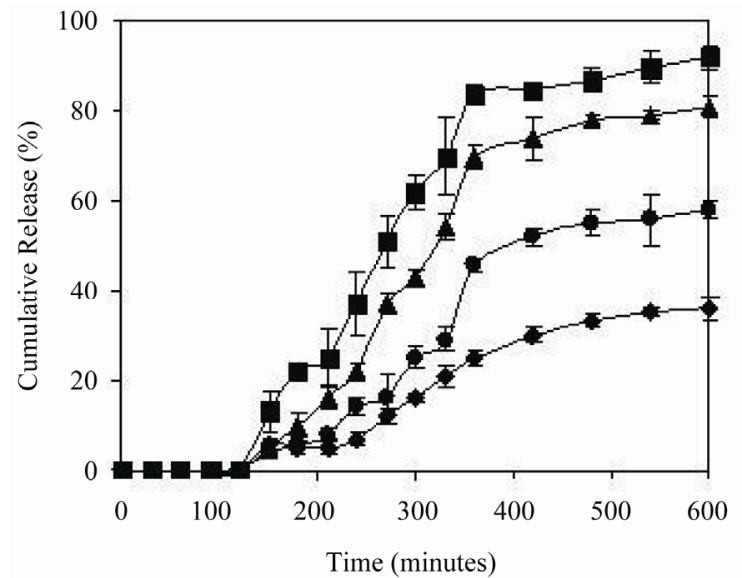

Figure 5. \% Cumulative release of NS from different IPN formulations loaded with $33 \%$ of drug at concentration of GA: $2.5 \%$ and exposure time to GA: 15 min. Symbols: A2 $(\bullet), \mathrm{B} 2(\Delta), \mathrm{C} 2(\bullet), \mathrm{D} 2(\bullet)$.

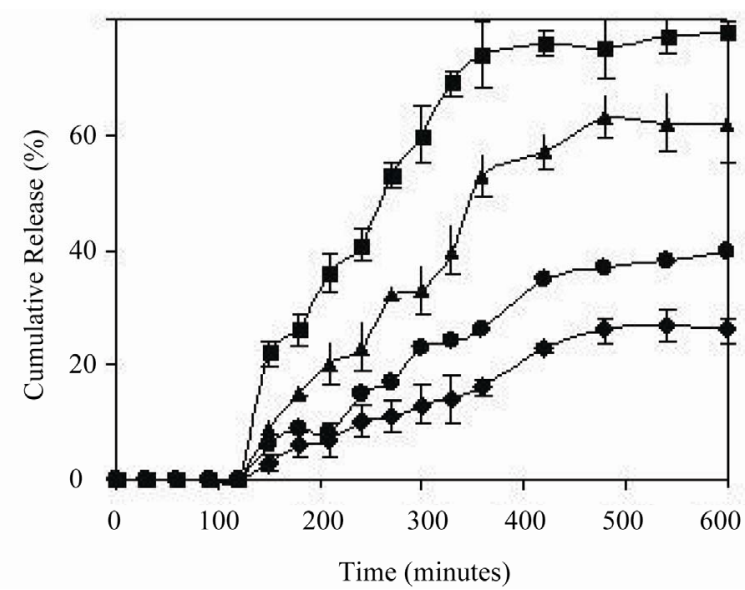

Figure 6. \% Cumulative release of NS from different IPN formulations loaded with $20 \%$ of drug at concentration of GA: $2.5 \%$ and exposure time to GA: 15 min. Symbols: A3 (匹), B3 ( $\Delta), \mathrm{C} 3(\bullet), \mathrm{D3}(\bullet)$.

Table 2. \% Equilibrium swelling degree for the empty IPNs at pH 7.4.

\begin{tabular}{cc}
\hline Formulation Code & Equilibrium swelling degree (\%) \\
\hline A & $117.83 \pm 4.78$ \\
B & $1043.40 \pm 3.45$ \\
C & $376.54 \pm 1.25$ \\
D & $157.36 \pm 1.43$ \\
\hline
\end{tabular}

It was seen from the figures that NaAlg microspheres produced nearly $92 \%$ cumulative drug release in $10 \mathrm{~h}$, whereas IPN microspheres produced up to $80 \%$ cumulative release at the end of the $10 \mathrm{~h}$. Alginate is a natural water-soluble polymer and contains hydroxyl and carboxyl groups, which impart hydrophilicity to the molecule. On the other hand PVA is virtually a linear polymer with a small hydrated volume compared to alginate and thus PVA produces a compact network of macromolecular chains in the IPN. The release rates of NS to an external medium are more difficult compared to the NaAlg microspheres. Similar results can be found from the published reports $[16,17]$. In the study of Krishna Rao and coworkers, controlled release of cefadroxil from IPN microspheres based on chitosan, acrylamide-graftedpoly(vinyl alcohol) and hydrolyzed acrylamide-graftedpoly(vinyl alcohol) were investigated. They have reported that the blend microgels have shown longer drug release rates than the plain chitosan microgels.

Effect of $33 \%$ (wt) NaAlg content in formulations B1, B2 and B3 on the release rates were presented in Figure 7. Also release studies were done for $50 \%(\mathrm{wt})$ and $66 \%$ (wt) NaAlg content and release results were shown in Figure 8 and Figure 9, respectively. The microspheres containing \% 50 NaAlg has the formulations given in 


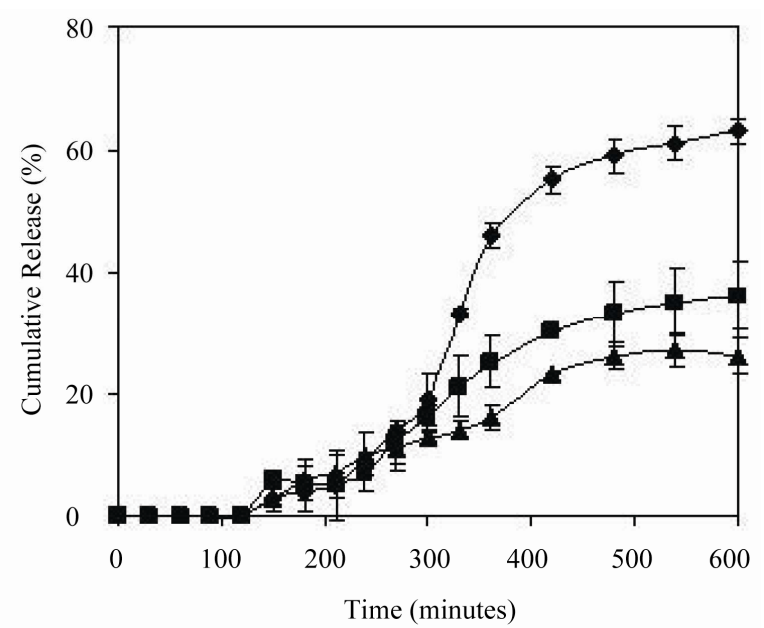

Figure 7. \% Cumulative release of NS from IPN microspheres containing $33 \%$ of $\mathrm{NaAlg}$ at concentration of GA: 2.5\% and exposure time to GA: 15 min. Symbols: B1 (४), B2 (匹), B3 ( $\mathbf{\Delta})$.

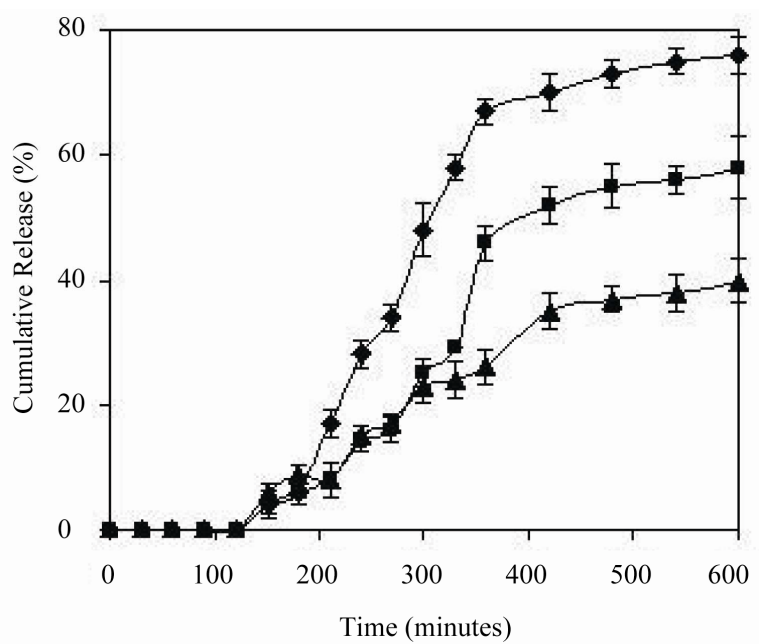

Figure 8. \% Cumulative release of NS from IPN microspheres containing $50 \%$ of $\mathrm{NaAlg}$ at concentration of GA: 2.5\% and exposure time to GA: $15 \mathrm{~min}$. Symbols: C1 (४), C2 (घ), C3 ( $\Delta)$.

Table 1 as C1, C2, C3 and the microspheres containing \% 66 NaAlg has the formulations given in Table 1 as D1, D2, D3.

Release results showed that formulations containing the highest amount of NS (50 wt\%) displayed higher release than those formulations containing low amount of NS. As the amount of drug increased from $20 \%$ to $50 \%$, the \% cumulative release rate increased from 26 to 84 . This is obvious that as the amount of NaAlg increases in the matrix, diffusion of NS occur faster and higher from the swollen IPN [17]. Ramesh Babu and coworkers prepared IPN microgels of sodium alginate-acrylic acid for the controlled release of ibuprofen. They reported that

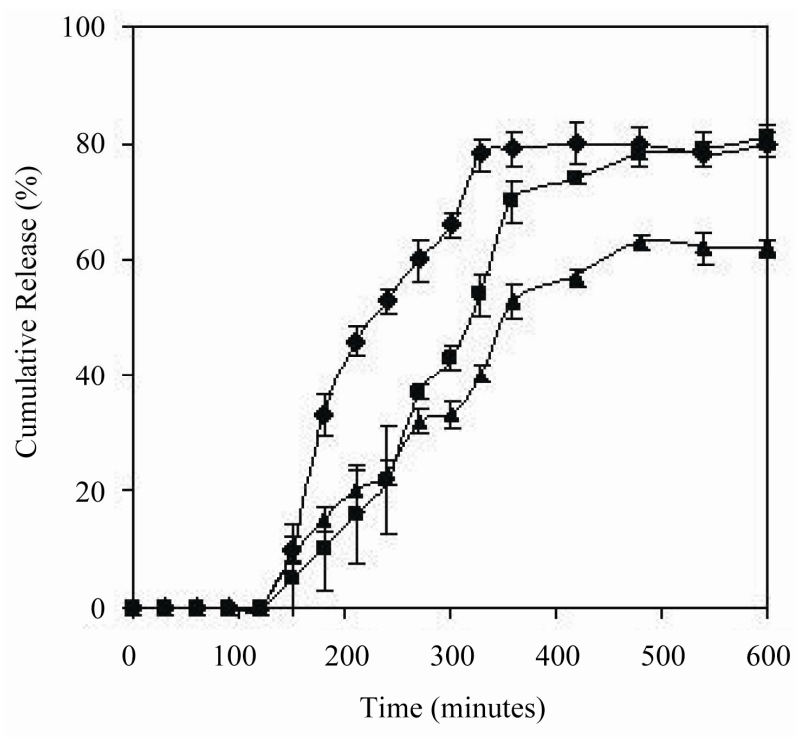

Figure 9. \% Cumulative release of NS from IPN microspheres containing $66 \%$ of $\mathrm{NaAlg}$ at concentration of GA: 2.5\% and exposure time to GA: $15 \mathrm{~min}$. Symbols: D1 (४), D2 (घ), D3 ( $\mathbf{\Delta})$.

formulations containing the highest amount of drug (75\%) displayed faster and higher release rates than those formulations containing a small amount of ibuprofen.

\subsection{Effect of Crosslinking Agent on the NS Release}

Varying exposure time of microspheres to GA at a fixed amount of the NS/polymer ratio (20 wt\%) are displayed in Figure 10 which clearly indicates that with increasing exposure time to GA (5 - $15 \mathrm{~min}$ ), the cumulative release decreases. Increasing exposure time to GA results in an increase in the crosslink density of the beads which gives rise to a compact network of macromolecular chains. As expected, the release of NS becomes slower at higher of GA, but becomes faster at lower amount of GA. The maximum NS release was obtained as $80 \%$ from the microspheres prepared with an exposure time of 5 minute.

To understand the extent of crosslinking of the polymer, it is necessary to calculate the molar mass $\left(\mathrm{M}_{\mathrm{C}}\right)$ between the crosslinks of the polymer. $\mathrm{M}_{\mathrm{C}}$ can be calculated from the equilibrium swelling volume of the polymer in a solvent [13].

Degree of crosslinking of polymer beads was calculated using Flory-Rehner equation as given below:

$$
M_{C}=-\delta_{p} V_{S} \phi^{1 / 3}\left[\ln (1-\phi)+\phi+\chi \phi^{2}\right]^{-1}
$$

$\phi$ is the volume fraction of the polymer in the swollen state and can be calculated as: 


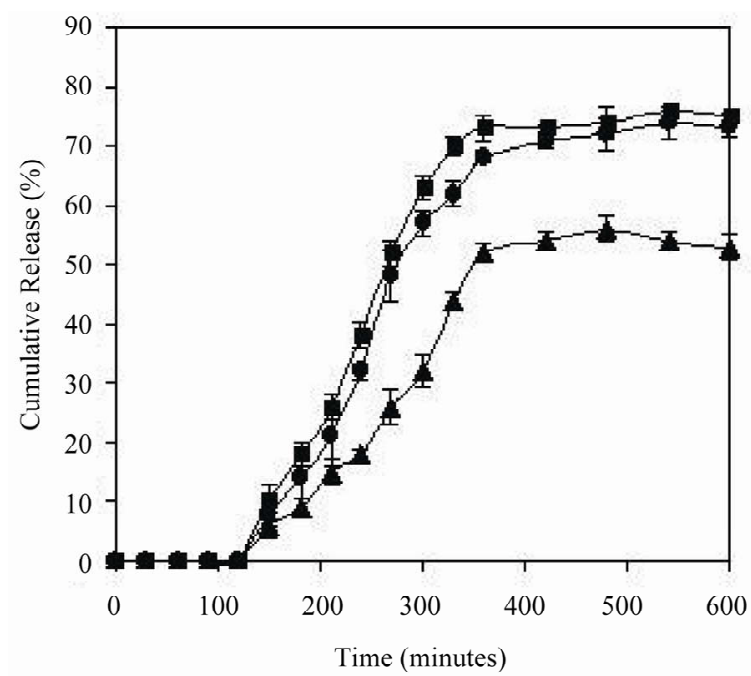

Figure 10. \% Cumulative release of NS from IPN microspheres containing different time of crosslinking agent. $66 \%$ NaAlg, 20\% NS, 25\% of GA. Symbols: 5 min (ø), 10 $\min (\diamond), 15 \min (\Delta)$.

$$
\phi=\left[1+\frac{\delta_{P}}{\delta_{S}}\left(\frac{M_{a}}{M_{b}}\right)-\frac{\delta_{P}}{\delta_{S}}\right]^{-1}
$$

where $\delta_{p}$ and $\delta_{S}$ are the densities of the polymer and solvent, respectively. $M_{a}$ and $M_{b}$ are the mass of the polymer before and after swelling, respectively. $V_{s}$ is the molar volume fraction of the polymer in the swollen state.

Interaction parameter $\chi$ can be calculated from the Flory-Rehner equation [18].

$$
\begin{aligned}
\chi= & {\left[\phi(1-\phi)^{-1}+N \ln (1-\phi)+N \phi\right] } \\
& \cdot\left[2 \phi-\phi^{2} N-\phi^{2} T^{-1}(\mathrm{~d} \phi / \mathrm{d} T)^{-1}\right]^{-1}
\end{aligned}
$$

where $N=\left(\phi^{2 / 3} / 3-2 / 3\right)\left(\phi^{1 / 3}-2 \phi / 3\right)^{-1}$ and temperature is taken as Kelvin.

Molar masses between crosslinking and polymer calculated for the NS loaded beads are presented in Table 3. As it is seen from Table 3 when the crosslinking of the polymer increased $M_{c}$ values decreased, since the network becomes more intensive structure. Also, the $M_{c}$ values increased with an increase in NaAlg content of the formulation, indicating an intensive structure.

\subsection{Analysis of Kinetic Results}

Solvent sorption by a microsphere depends mechanistically on the diffusion of water molecules into the gel matrix and subsequent relaxation of macromolecular chains of the microsphere [19]. The release data of all the systems have been further substantiated by fitting the fraction release data $M_{t} / M_{\infty}$ to an empirical equation proposed by Peppas [20].

$$
k t^{n}=\frac{M_{t}}{M_{\infty}}
$$

In the equation, $M_{t}$ is the amount of NS released at time t and $M_{\infty}$ is the drug released at equilibrium time; $\mathrm{k}$, a constant characteristic of the drug-polymer system; and $\mathrm{n}$, the diffusional exponent which suggests the nature of the release mechanism. Fickian release is defined by initial $t^{1 / 2}$ time dependence of the fractional release for slabs, cylinders and spheres. Analogously Case-II trans-

Table 3. Values of $M_{C}, k, n, r$ for NS containing IPNs.

\begin{tabular}{cccccc}
\hline Formulation Code & $\mathrm{k}\left(\mathrm{min}^{-\mathrm{n}}\right) \times 10^{2}$ & $\mathrm{n}$ & $\mathrm{r}$ & $\mathrm{M}_{\mathrm{C}}$ & Diffusion Mechanism \\
\hline A1 & 0.0087 & 0.8765 & 0.9793 & 1492 & Anomalous Transport \\
A2 & 0.0180 & 0.7475 & 0.9862 & 1597 & Anomalous Transport \\
A3 & 0.0230 & 0.6983 & 0.9756 & 1671 & Anomalous Transport \\
A4 & 0.0189 & 0.6538 & 0.9774 & 1748 & Anomalous Transport \\
A5 & 0.0257 & 0.6578 & 0.9865 & 1886 & Anomalous Transport \\
B1 & 0.0009 & 0.9064 & 0.9917 & 2596 & Anomalous Transport \\
B2 & 0.0008 & 0.1013 & 0.9987 & 2547 & Case II \\
B3 & 0.0031 & 0.8865 & 0.9946 & 2508 & Anomalous Transport \\
C1 & 0.0008 & 1.0402 & 0.9980 & 1986 & Case II \\
C2 & 0.0006 & 1.1657 & 0.9959 & 2067 & Case II \\
C3 & 0.0042 & 0.7852 & 0.9947 & 2247 & Anomalous Transport \\
D1 & 0.0005 & 1.2415 & 0.9967 & 1954 & Case II \\
D2 & 0.0008 & 1.1357 & 0.9948 & 1941 & Case II \\
D3 & 0.0004 & 1.3475 & 0.9972 & 1983 & Case II
\end{tabular}


port is defined by an initial linear time dependence of the fractional release for all geometries [21]. A value of $n$; 0.5 indicates the Fickian transport (mechanism), while n; 1 is of Case II or non-Fickian transport (swelling controlled). The intermediary values ranging between 0.5 and 1.0 are indicative of the anomalous transport. The least squares estimations of the fractional release data along with the estimated correlation coefficient values, $r$, are presented in Table 3. From these data, the $\mathrm{n}$ value ranged between 0.6538 - 1.3475, indicating that, NS from the microspheres slightly deviates from the Fickian transport.

\section{Conclusions}

This work demonstrates the effective encapsulation of NS into NaAlg and PVA to produce IPN microspheres by emulsification crosslinking method. The IPN microspheres demonstrated better controlled release results than pure NaAlg, indicating the suitability of IPN for microsphere preparation. The crosslink density was signicantly affected by the amount of GA and the polymers in the formulations. The release of NS was found to be dependent on the extent of crosslinking, the amount of drug loading and the polymer content of the matrix. The release mechanism showed a slight deviation from the Fickian behavior. It can be concluded that microspheres prepared in this study can be effectively used as a controlled release device for the release of NS.

\section{Acknowledgements}

The author is grateful to the Gazi University Scientific Research Foundation for support of this study and to Novartis Company for the supply of the drug (Naproxen Sodium).

\section{REFERENCES}

[1] O. C. Farokhzad and R. Langer, "Impact of Nanotechnology on Drug Delivery,” ACS Nano, Vol. 3, No. 1, 2009, pp. 16-20. doi:10.1021/nn900002m

[2] D. A. Lavan, T. McGuire and R. Langer, "Small-Scale Systems for in Vivo Drug Delivery,” Nature Biotechnology, Vol. 21, No. 10, 2003, pp. 1184-1191. doi:10.1038/nbt876

[3] G. M. Whitesides, “The 'Right' Size in Nanobiotechnology,” Nature Biotechnology, Vol. 21, No. 10, 2003, pp. 1161- 1165. doi:10.1038/nbt872

[4] K. Srenivasan, "On the Restriction of the Release of Water-Soluble Component from Poly Vinyl Alcohol Film by Blending B-Cyclodextrin,” Journal of Applied Polymer Science, Vol. 65, No. 9, 1997, pp. 1829-1832. doi:10.1002/(SICI)1097-4628(19970829)65:9<1829::AI D-APP20>3.0.CO;2-G

[5] S. Y. Kim and Y. M. Lee, "Drug Release Behavior of Electrical Responsive Poly(vinyl alcohol)/Poly(acrylic acid) IPN Hydrogels under an Electric Stimulus,” Journal of Applied Polymer Science, Vol. 74, No. 7, 1999, pp. 1752-1761. doi:10.1002/(SICI)1097-4628(19991114)74:7<1752::AI D-APP18>3.0.CO;2-H

[6] D. K. Kweon and D. W. Kang, "Drug Release Behavior of Chitosan-G-Poly(vinyl alcohol) Copolymer Matrix,” Journal of Applied Polymer Science, Vol. 74, No. 2, 1999, pp. 458-464.

doi:10.1002/(SICI)1097-4628(19991010)74:2<458::AIDAPP29>3.0.CO;2-6

[7] A. B. Pepperman and J. C. W. Kuan, "Controlled Release Formulations of Alachlor Based on Calcium Alginate," Journal of Controlled Release, Vol. 34, No. 1, 1995, pp. 17-23. doi:10.1016/0168-3659(94)00111-7

[8] S. G. Kumbar and T. M. Aminabhavi, "Preparation and Characterization of Interpenetrating Network Beads of Poly(vinyl alcohol)-G-Poly(acrylamide) with Sodium Alginate and Their Controlled Release Characteristics for Cypermethrin Pesticide," Journal of Applied Polymer Science, Vol. 84, No. 3, 2002, pp. 552-560. doi:10.1002/app.10306

[9] S. J. Kim, S. G. Yoon and S. I. Kim, "Synthesis and Characteristics of Interpenetrating Polymer Network Hydrogels Composed of Alginate and Poly(diallydimethylammonium chloride)”. Journal of Applied Polymer Science, Vol. 91, No. 6, 2004, pp. 3705-3709. doi:10.1002/app.13615

[10] M. Fernandez-Perez, E. Gonzales-Pradas, M. VillafrancaSanchez and F. Flores-Cespedes, "Mobility of Isoproturon from an Alginate \pm Bentonite Controlled Release Formulation in Layered Soil," Chemosphere, Vol. 41, No. 9, 2000, pp. 1495-1501. doi:10.1016/S0045-6535(99)00516-0

[11] V. Pillay and R. Fassihi, "In Vitro Release Modulation from Crosslinked Pellets for Site-Specific Drug Delivery to the Gastrointestinal Tract I. Comparison of pH-Responsive Drug Release and Associated Kinetics,” Journal of Controlled Release, Vol. 59, No. 2, 1999, pp. 229-242. doi:10.1016/S0168-3659(98)00196-5

[12] X. Z. Shu and K. Zhu, "The Release Behavior of Brilliant Blue from Calcium-Alginate Gel Beads Coated by Chitosan: The Preparation Method Effect," European Journal of Pharmaceutics and Biopharmaceutics, Vol. 53, No. 2, 2002, pp. 193-201. doi:10.1016/S0939-6411(01)00247-8

[13] A. R. Kulkarni, K. S. Soppimath, T. M. Aminabhavi, A. M. Dave and M. H. Mehta, "Gluteraldehyde Crosslinked Sodium Alginate Beads Containing Liquid Pesticide for Soil Application,” Journal of Controlled Release, Vol. 63, No. 1-2, 2000, pp. 97-105. doi:10.1016/S0168-3659(99)00176-5

[14] S. G. Adoor, B. Prathab, L. S. Manjeshwar and T. M. Aminabhavi, "Mixed Matrix Membranes of SodiumAlginate and Poly(vinyl alcohol) for Pervaporation Dehydration of Isopropanol at Different Temperatures," Polymer, Vol. 48, No. 18, 2007, pp. 5417-5430.

doi:10.1016/j.polymer.2007.06.064 
[15] A. P. Rokhade, N. B. Shelke, S. A. Patil and T. M. Aminabhavi, "Novel Interpenetrating Polymer Network Microspheres of Chitosan and Methylcellulose for Controlled Release of Theophylline,” Carbohydrate Polymers, Vol. 69, No. 4, 2007, pp. 678-687. doi:10.1016/j.carbpol.2007.02.008

[16] K. S. V. Krishna-Rao, B. V. Kumar Naidu, M. C. S. Subha, M. Sairam and T. M. Aminabhavi, "Novel Chitosan-Based pH-Sensitive Interpenetrating Network Microgels for the Controlled Release of Cefadroxil," Carbohydrate Polymers, Vol. 66, No. 3, 2006, pp. 333-344. doi:10.1016/j.carbpol.2006.03.025

[17] V. Ramesh Babu, K. S. V. Krishna-Rao, M. Sairam, B. Vijaya-Kumar-Naidu, K. M. Hosamani and T. M. Aminabhavi, "pH Sensitive Interpenetrating Network Microgels of Sodium Alginate-Acrylic Acid for the Controlled Release of Ibuprofen,” Journal of Applied Polymer Science, Vol. 99, No. 5, 2006, pp. 2671-2678.

\section{doi:10.1002/app.22760}

[18] P. J. Flory, "Principles of Polymer Chemistry," Cornell University Press, New York, 1953.

[19] A. K. Bajpai and M. Sharma, "Preparation and Characterization of Binary Grafted Polymeric Blends of Polyvinyl Alcohol and Gelatin and Evaluation of their Water Uptake Potential,” Journal of Macromolecular Science A, Vol. 42, 2005, pp. 663-682.

[20] N. A. Peppas, "Analysis of Fickian non-Fickian Drug Release from polymers". Pharmaceutica Acta Helvetiae, Vol. 60, No. 4, 1985 pp. 110-111.

[21] P. L. Ritger and N. A. Peppas, "A simple Equation for Description of Solute Release II. Fickian and Anomalous Release from Swellable Devices," Journal of Controlled Release, Vol. 5, No. 1, 1987 pp. 37-42. doi:10.1016/0168-3659(87)90035-6 